\title{
Avaliação da concentração de mercúrio em sedimentos e material particulado no rio Acre, estado do Acre, Brasil.
}

\author{
Artur Fernando Silva MASCARENHAS ${ }^{1}$, Edilson da Silva BRABO ${ }^{2}$, Alexandre Pessoa da SILVA ${ }^{3}$, \\ Kleber de Freitas FAYAL ${ }^{2}$, Iracina Maura de JESUS ${ }^{2}$, Elisabeth C. de Oliveira SANTOS ${ }^{2}$
}

\begin{abstract}
RESUMO
A avaliação dos teores de mercúrio em sistemas aquáticos sem influência direta de fontes antropogênicas conhecidas não tem sido conduzida com freqüência na região Amazônica. Visando contribuir para esclarecer a ocorrência de valores elevados de $\mathrm{Hg}$ em peixes consumidos pela população de Rio Branco - AC, o Instituto Evandro Chagas - IEC, realizou um estudo para quantificar os teores de $\mathrm{Hg}$ em sedimentos de fundo e material particulado no rio Acre e alguns afluentes, além da caracterização físico-química das águas entre as cidades de Brasiléia e Assis Brasil. As amostras de sedimentos foram peneiradas na fração $<250$ mesh e o material particulado obtido por floculação com $\mathrm{Al}_{2} \mathrm{SO}_{4}$. Uma massa de $250 \mathrm{mg}$ dos materiais foram submetidos a digestão ácida e as determinações de $\mathrm{Hg}$ realizadas por Espectrofotometria de Absorção Atômica, com geração de vapor frio. Os parâmetros físico-químicos pH, condutividade elétrica, temperatura e sólidos totais dissolvidos, foram feitos no campo, por métodos potenciométricos. Os teores de $\mathrm{Hg}$ nos sedimentos de fundo variaram entre 0,018 e $0,184 \mu \mathrm{g} \mathrm{g}^{-1}$, com média de $0,054 \pm 0,034 \mu \mathrm{g} \mathrm{g}^{-1}$, enquanto que no material particulado a variação foi de 0,067 a $0,220 \mu \mathrm{g} \mathrm{g}^{-1} \mathrm{e}$ média de $0,098 \pm 0,037 \mu \mathrm{g} \mathrm{g}^{-1}$. As águas possuem características levemente ácidas indicadas pelos valores de $\mathrm{pH}$ que variaram entre 5,80 - 6,95. A condutividade elétrica variou de $151,60-1.151,00 \mu \mathrm{S} \mathrm{cm}^{-1}$. Os teores de $\mathrm{Hg}$ nos materiais analisados encontram-se dentro da faixa dos valores observados para os rios amazônicos "não poluídos". Entretanto, estudos complementares deverão ser implementados para elucidar a origem e os processos de biodisponibilidade do mercúrio.
\end{abstract}

\section{PALAVRAS-CHAVE}

Amazônia, mercúrio, sedimentos, material particulado, rio Acre.

\section{Mercury concentration assessment in botton sediments and suspended solids from the Acre river, in the State of Acre, Brazil.}

\begin{abstract}
Mercury level assessment in aquatic system areas without direct influence from antropogenic sources bave not been well studied in the Amazon region. For the identification of the origin of high values of Hg in fish consumed by the population of Rio Branco - AC, the Evandro Chagas Institute - IEC, studied the mercury levels in sediments, suspended solids and the physical-chemical characterization of waters, in the Acre river and also some in affluents, between the cities of Brasiléia and Assis Brasil. Bottom sediment samples were sieved to $<250$ mesh fraction after drying. The suspended solid was obtained by precipitation with $\mathrm{Al}_{2} \mathrm{SO}_{4}$ About $250 \mathrm{mg}$ of the material was submitted to acid digestion and the determinations of $\mathrm{Hg}$ were made by Could Vapor Atomic Absorption Spectrometry. The physical-chemical parameters $p H$, electric conductivity, temperature and dissolved total solids, were studied in the field, by potenciometric methods. The Hg levels in bottom sediments range between 0,018 and $0,184 \mu \mathrm{g} \mathrm{g}{ }^{-1}$, mean of $0,054 \pm 0,034 \mu \mathrm{g} \mathrm{g}{ }^{1}$, while the suspended solids varied between 0,067 and $0,220 \mu \mathrm{g} \mathrm{g}$, average of $0,098 \pm 0,037 \mu \mathrm{g} \mathrm{g}^{-1}$. The waters were slightly acid with pH varying among 5,80 - 6,95 and conductivity electric 151,60-1.151,00 $\mu \mathrm{S} \mathrm{cm} \mathrm{cm}^{-1}$. The mercury levels in the analyzed materials was below standard levels for the non-polluted Amazon rivers. However complementary studies should be developed to elucidate the origin and the processes of mercury biodisponibility.
\end{abstract}

\section{KEY WORDS}

Amazonia, mercury, bottom sediments, suspended solids, Acre river.

\footnotetext{
1.Secretaria Executiva de Indústria, Comércio e Mineração - SEICOM. Av. Presidente Vargas, no 1020, Centro, CEP 66.017-000, Belém - Pará, Brasil, fone: (0xx) 91 241-18-18, fax: (0xx) 91 223-26-89, e-mail: masca@amazon.com.br

2.MS / SVS / Instituto Evandro Chagas / Seção de Meio Ambiente - SAMAM. Av. Almirante Barroso, 492, CEP 66.000-000 Belém - Pará, Brasil, fone/fax : (0xx) 91 255-20-03, e-mail: coehma@amazon.com.br

3. AMBIOS Engenharia e Processos Ltda. Rua Califórnia nº 792 - CEP 04566-061 - Brooklin - São Paulo - SP - e-mail: contato@ambios.com.br
} 


\section{ACTA \\ AMAZONICA}

AVALIAÇÃO DA CONCENTRAÇÃO DE MERCÚRIO EM SEDIMENTOS

E MATERIAL PARTICULADO NO RIO ACRE, ESTADO DO ACRE, BRASIL

\section{INTRODUÇÃO}

A presença de mercúrio $(\mathrm{Hg})$ na região Amazônica têm sido associada a mineração artesanal, conhecida como "garimpagem", onde este metal na forma líquida é utilizado para recuperação de partículas de ouro com granulometria fina (Martinelli et al., 1988; Pfeiffer \& Lacerda, 1988; Pfeiffer et al., 1989; Malm et al., 1990).

Vários estudos têm mostrado não só a contaminação mercurial dos ecossistemas amazônicos onde essa atividade é desenvolvida, como os seus efeitos sobre a saúde das populações (Silva, 1993a; Mascarenhas et al., 1998; Santos et al., 1998; Brabo et al.,1998; Brabo et al., 1999; Santos, 1993; Barbosa et al., 1995; Akagi et al., 1995; Lebel et al., 1997; Jesus et al., 1998).

As informações sobre as quantidades de mercúrio utilizadas na atividade garimpeira e lançadas ao meio ambiente amazônico são extremamente contraditórias. Entre as estimativas mais citadas reportam-se valores da ordem de uma a duas centenas de toneladas de $\mathrm{Hg}$ /ano nas últimas duas décadas. Deste total, cerca de $50 \%$ são liberadas para os cursos d'água durante o processo de extração e recuperação, e o restante para a atmosfera durante os procedimentos de queima do amálgama (Pfeiffer \& Lacerda, 1988; Pfeiffer et al.; 1989; Rodrigues et al., 1994; Malm et al., 1995; Hacon et al., 1997).

No início da década de 90 estudos realizados por Veiga et al. (1994), sobre a presença de $\mathrm{Hg}$ no ambiente amazônico mostraram uma associação entre a queima de biomassa florestal e a quantidade de $\mathrm{Hg}$ lançadas para a atmosfera anualmente, estimada em 90 ton/ano. Segundo estes autores este fato é responsável por uma parcela importante de $\mathrm{Hg}$ encontrada nos ecossistemas aquáticos.

Outros estudos, entretanto, têm relacionado à presença de mercúrio em áreas sem influência direta da garimpagem aos processos de erosão e lixiviação das partículas que contém mercúrio associado aos óxidos e hidróxidos de Ferro, favorecidos pelos desmatamentos, os quais promovem a exposição dos solos. Estes materiais têm sido apontados como fontes naturais de mercúrio para os ecossistemas aquáticos, disponibilizando-o no meio aquático para os processos de metilação e acumulação na biota ( Roulet \& Lucotte, 1995; Roulet et al., 1998; Fostier et al., 1999; Ribeiro et al., 1999, Zeidemann et al., 1999).

Este estudo faz parte de um programa de trabalho do Instituto Evandro Chagas / SVS sobre a ocorrência de Hg em áreas sem registro de ação antrópica emissora de $\mathrm{Hg}$ na região amazônica, e foi conduzido em função da necessidade de elucidação da presença de níveis elevados de Hg em amostras de pescado procedentes do Estado do Acre, coletados/ capturados dos rios Acre e Purús que mostraram teores bem acima de $0,500 \mu \mathrm{g} \mathrm{g}^{-1} \mathrm{de} \mathrm{Hg}$ (peso úmido), valor recomendado pela OMS para consumo humano (Santos et al., 2002). Por outro lado, a inexistência na região, de atividades humanas emissoras do poluente como, por exemplo, o garimpo, levanta uma série de dúvidas quanto a fonte de $\mathrm{Hg}$ para a biota. Este estudo propôs avaliar a distribuição dos teores de $\mathrm{Hg}$ nos sedimentos de fundo e material particulado no rio Acre entre as cidades de Brasiléia e Assis Brasil no Estado do Acre e seu potencial de acumulação e liberação de $\mathrm{Hg}$ para a biota.

\section{MATERIAL E MÉTODOS}

\section{Área de Estudo}

A área de estudo está localizada na bacia do rio Acre, no trecho entre as cidades de Brasiléia e Assis Brasil, área de fronteira entre o Brasil, Peru e Bolívia (Figura 1).

O substrato geológico é representado pela Formação Solimões, que possui uma ampla distribuição territorial, abrangendo praticamente a totalidade da bacia hidrográfica do rio Solimões. Esta unidade é constituída principalmente por arenitos quartzosos e arcoseanos, intercalados ou interdigitados com siltitos e argilitos sílticos, localmente calcíferos, com lentes e concreções calcárias, gipsíferas e limoníticas. Ocasionalmente encontram-se níveis ou lentes com matéria orgânica (turfa e linhito), além de arenitos ferruginosos e concrecionados, conglomerados e brechas. A Formação Solimões apresenta características de ambiente fluvial e fluvio-lacustre depositados entre os períodos Plioceno e Pleistoceno Superior (FIBGE, 1990). Encontrase, ainda, a presença de fósseis, principalmente de vertebrados de médio e grande porte, destacando-se os quelônios, crocodilídios e toxodontes (Costa et al. 1989).

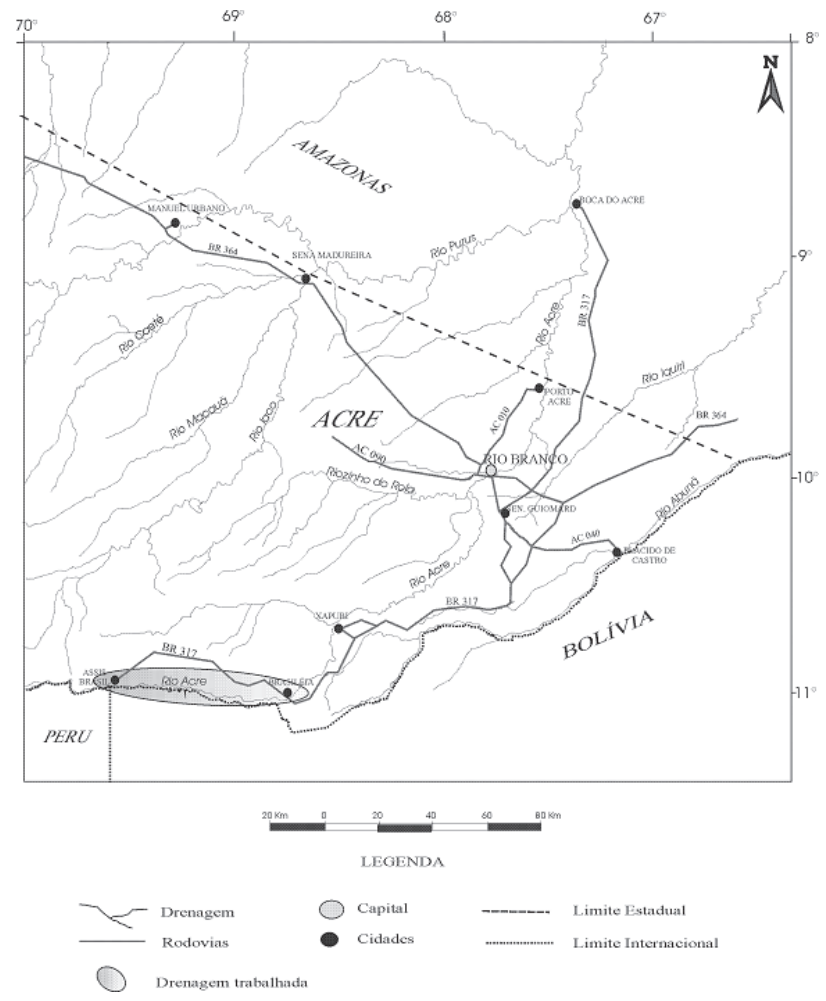

Figura 1 - Mapa da região de estudo entre Brasiléia e Assis Brasil, Estado do Acre. 


\section{ACTA AMAZONICA}

\section{Procedimentos de Campo}

A campanha foi realizada no rio Acre entre as cidades de Brasiléia e Assis Brasil no período de 3 a 12 de novembro de 1998, em 32 pontos de amostragem, durante o período de seca no Estado do Acre (Figura 2).

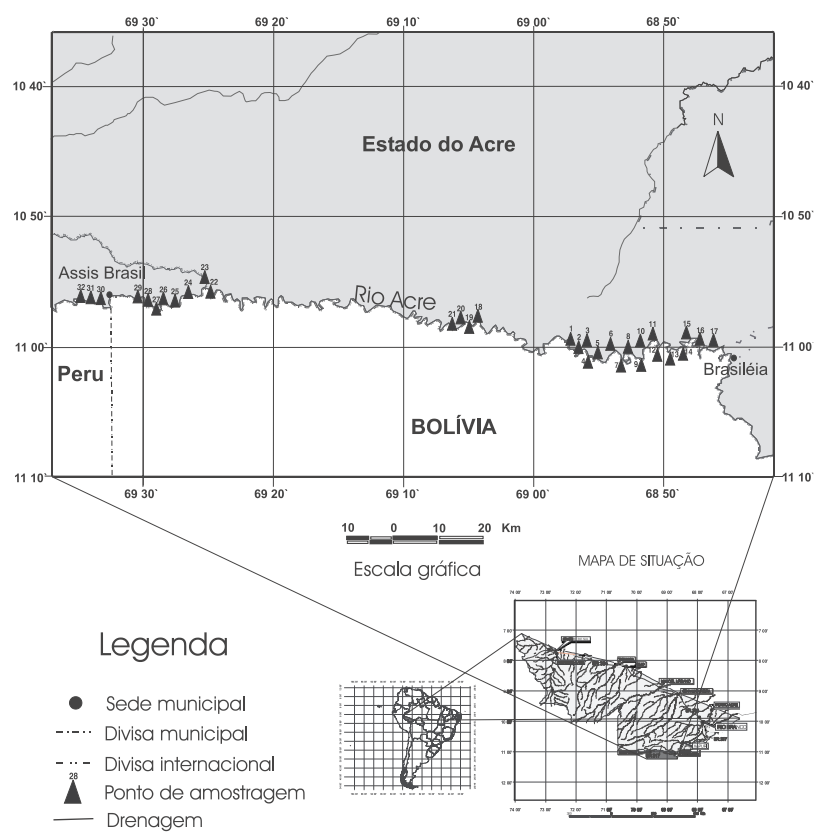

Figura 2 - Mapa de localização dos pontos de amostragem.

\section{Sedimentos de Fundo}

As amostras de sedimentos foram coletadas preferencialmente nos locais de baixa profundidade e menor velocidade de fluxo, onde ocorre a sedimentação do material carreado pela drenagem. O material é constituído de argilas, siltes e areias finas, de coloração variada cinza a marrom avermelhada, com pouca matéria orgânica. As amostras foram coletadas com o auxílio de uma pá de plástico, em uma área de $4 \mathrm{~m}$ de diâmetro, a partir de um ponto, foram coletadas varias alíquotas até atingir aproximadamente $1 \mathrm{~kg}$ de material da porção mais superficial do leito das drenagens (Silva, 1993b). O material foi acondicionado em sacos plásticos, identificados e mantidos sob refrigeração.

\section{Material Particulado em Suspensão}

O material particulado em suspensão (MPS) foi obtido de acordo com a metodologia proposta por Silva et al. (1993), baseada na floculação e precipitação deste material com sulfato de alumínio. Um volume de cinco litros de água foi coletado em recipiente de polietileno, previamente lavado com $\mathrm{HNO} 310 \%$ e água destilada e deionizada Milli$\mathrm{Q}$, e adicionado um volume de $10 \mathrm{ml}$ da solução de $\mathrm{Al}_{2}\left(\mathrm{SO}_{4}\right)_{3}$ a $10 \%$, seguido de agitação vigorosa. Após esta etapa, o pH da solução foi ajustado com $\mathrm{NaOH} 1 \mathrm{~N}$, até pH 7. Após nova agitação, a solução foi mantida em repouso por 30 minutos e retirou-se o excesso de sobrenadante, desprezando-o, sendo o precipitado acondicionado em frascos de fundo cônico de $50 \mathrm{~mL}$ e mantido sob refrigeração.

\section{Procedimentos de Laboratório}

Preparação das amostras para análise de $\mathrm{Hg}$

No laboratório as amostras de sedimentos de fundo foram secas a temperatura ambiente, protegidas de contaminação externa, desagregadas, peneiradas com malha de aço inoxidável, utilizando-se agitador magnético de peneiras marca Bertel, separando-se a fração menor que 250 mesh.

As amostras de material particulado, acondicionadas nos tubos cônicos de $50 \mathrm{~mL}$ foram centrifugadas a $2.000 \mathrm{rpm}$, durante 10 minutos e a fase líquida remanescente retirada por sucção com o auxílio de um aspirador. A fase sólida foi secada à vácuo, com o auxílio de um dessecador para os procedimentos analíticos posteriores.

\section{Análise de Mercúrio Total}

As vidrarias utilizadas nos procedimentos analíticos foram previamente mantidas em solução de $\mathrm{HNO}_{3} 10 \%$, por $24 \mathrm{~h}, \mathrm{KMnO}_{4}$ em solução $\mathrm{H}_{2} \mathrm{SO}_{4} 1 \mathrm{~N}$ e $\mathrm{NH}_{2} \mathrm{OH} \cdot \mathrm{HCl}$ a $10 \%$, lavados com água corrente, e posteriormente lavados com água destilada e deionizada em um sistema Milli-Q e secos em estufa à temperatura de $50^{\circ} \mathrm{C}$.

Para as determinações de $\mathrm{Hg}$ total, as amostras foram submetidas aos procedimentos analíticos pelo método proposto por Akagi et al. (1996) e descrito resumidamente a seguir: uma massa de $250 \mathrm{mg}$ foi pesada e acondicionada em frascos de digestão de aproximadamente $50 \mathrm{~mL}$. Em seguida, $2 \mathrm{~mL}$ de $\mathrm{HNO}_{3}+\mathrm{HClO}_{4}(1: 1), 5 \mathrm{~mL}$ de $\mathrm{H}_{2} \mathrm{SO}_{4}, 1$ $\mathrm{mL}$ de $\mathrm{H}_{2} \mathrm{O}$ deionizada, foram adicionados. Após agitação, os frascos de digestão foram levados à chapa aquecedora à temperatura de $220{ }^{\circ} \mathrm{C}$ durante 20 minutos. Em seguida, as soluções foram resfriadas à temperatura ambiente, transferidas para balão volumétrico de $50 \mathrm{~mL}$, aferidas e homogeneizadas com água. Na realização das análises foram utilizados reagentes da marca Merck, suprapuro.

As determinações de $\mathrm{Hg}$ nas amostras foram feitas por Espectrofotometria de Absorção Atômica com sistema de geração de vapor a frio, utilizando-se o modelo Automatic Mercury Analyzer HG-3500 do fabricante K.K Sanso S.S, cujo princípio de funcionamento é mostrado na Figura 3. Durante a realização das análises foi construída uma curva de calibração das soluções padrões de 0,$0 ; 0,4 ; 0,8$ e $1,2 \mathrm{ng} \mathrm{mL}^{-1}$ e foram realizadas também a leitura de branco dos reagentes e de amostra certificada SRM $2710\left(32 \pm 5 \mu \mathrm{g} \mathrm{g}^{-1}\right)$, com recuperação de 95,0 a 98,5\%. As leituras das amostras foram realizadas em duplicatas com um desvio padrão médio de \pm 0,690 entre as réplicas. O limite de detecção do método é de $1 \mathrm{ng}$ de $\mathrm{Hg}$ absoluto. 


\section{ACTA AMAZONICA}

AVALIAÇÃO DA CONCENTRAÇÃO DE MERCÚRIO EM SEDIMENTOS

E MATERIAL PARTICULADO NO RIO ACRE, ESTADO DO ACRE, BRASIL

\section{Parâmetros físico-químicos}

As determinações físico-químicas foram realizadas no campo através de métodos potenciométricos. Os valores de $\mathrm{pH}$ foram obtidos utilizando-se $\mathrm{pH}$-metro da firma Schott Gerate, modelo CG-838, e os valores de condutividade elétrica, sólidos totais dissolvidos e temperatura foram determinados com sensores combinados, da firma Water Proof CE, modelo 4.200.

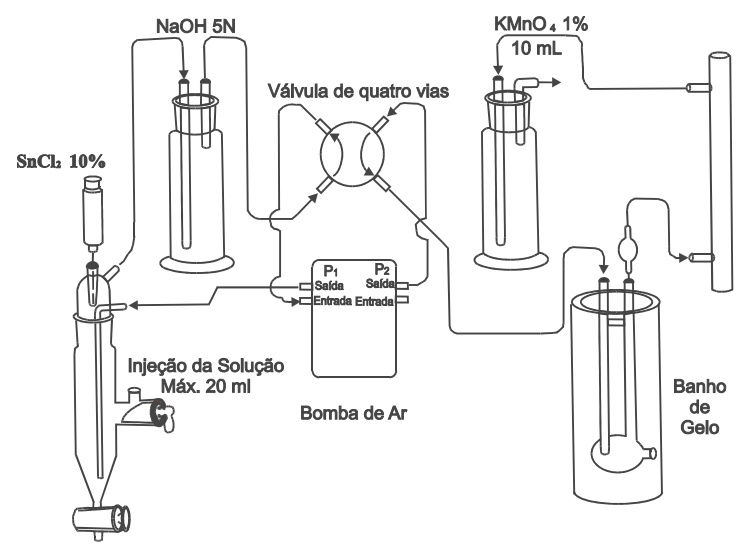

Figura 3 - Diagrama esquemático do princípio de funcionamento do MERCURY ANALIZER HG-3500.

\section{RESULTADOS}

\section{Teores de Hg em Sedimentos e no Material Particulado Suspenso}

Os resultados das medições dos parâmetros físico-químicos, os teores medidos de $\mathrm{Hg}$ nos sedimentos de fundo e no material particulado suspenso são mostrados na Tabela 1 .

Os resultados analíticos de $\mathrm{Hg}$ nos sedimentos de fundo $(n=32)$ não apresentaram uma variação significativa ao longo do trecho estudado. No rio Acre os teores de $\mathrm{Hg}$ nestes materiais variaram entre 0,018 e $0,184 \mu \mathrm{g} \mathrm{g}^{-1}$, com média de $0,058 \mu \mathrm{g} \mathrm{g}^{-1}$. Estes valores mostraram uma distribuição de $\mathrm{Hg}$ homogênea na área, indicando a ausência de fontes pontuais de Hg. Um comportamento similar foi observado nos afluentes do rio Acre, onde os valores de $\mathrm{Hg}$ variaram entre 0,027 e $0,078 \mu \mathrm{g} \mathrm{g}^{-1}$ com média de $0,048 \mu \mathrm{g} \mathrm{g}^{-1}$.

Os teores de $\mathrm{Hg}$ no material particulado suspenso foram avaliados nos mesmos locais de coleta de sedimentos de fundo, sendo que em apenas 21 pontos foi possível obter-se material suficiente para leitura. No rio Acre os valores variaram entre 0,067 e $0,088 \mu \mathrm{g} \mathrm{g}^{-1}$, com média de $0,076 \mu \mathrm{g} \mathrm{g}^{-1}$ (Tabela 1 ). Nos afluentes do rio Acre, verificou-se um aumento no valor médio de $\mathrm{Hg}$ no MPS que alcançou $0,116 \mu \mathrm{g} \mathrm{g}^{-1}$, variando entre 0,068 e 0,220 $\mu \mathrm{g} \mathrm{g}^{-1}$ (Tabela 1 e Figura 4).

Tabela 1 - Valores e médias de mercúrio e dos parâmetros físico-químicos obtidos no rio Acre e seua afluentes

\begin{tabular}{|c|c|c|c|c|c|c|c|}
\hline \multirow{3}{*}{ Pontos } & \multirow{3}{*}{ Localização } & \multirow{2}{*}{\multicolumn{4}{|c|}{$\mathrm{H}_{2} \mathrm{O}$}} & \multicolumn{2}{|c|}{$\mathrm{Hg}$} \\
\hline & & & & & & \multirow[t]{2}{*}{$\begin{array}{l}\text { Sed. } \\
\mu \mathrm{g} \mathrm{g}^{-1}\end{array}$} & \multirow[t]{2}{*}{$\begin{array}{l}\text { Mat. Part. } \\
\mu \mathrm{g} \mathrm{g}^{-1}\end{array}$} \\
\hline & & $\mathrm{PH}$ & $\begin{array}{l}\text { Cond. } \\
\mu S \mathrm{~cm}^{-1}\end{array}$ & $\begin{array}{c}\text { STD } \\
\mathrm{mg} \mathrm{L}^{-1}\end{array}$ & $\underset{{ }^{\circ} \mathrm{C}}{\text { Temp }}$ & & \\
\hline 1 & Ig. Ponte Grande (Rio Acre) & 5,80 & 151,6 & 91,0 & 25,8 & 0,027 & 0,112 \\
\hline 2 & Praia do Quixadá (Rio Acre) & 6,75 & 427,0 & 256,0 & 29 & 0,184 & 0,079 \\
\hline 3 & Ig. Quixadá (Rio Acre) & 6,75 & 492,0 & 294,0 & 28,1 & 0,046 & 0,131 \\
\hline 4 & Rio Acre (Colônia Espirito Santo) & 6,93 & 430,0 & 258,0 & 28,4 & 0,018 & * \\
\hline 5 & Rio Acre ( Colônia Espirito Santo) & \# & \# & $\#$ & $\#$ & 0,034 & * \\
\hline 6 & Ig. Santa Cruz (Rio Acre) & \# & 264,0 & 160,9 & 26,9 & 0,032 & 0,080 \\
\hline 7 & Ig. Santana (Rio Acre) & 6,65 & 332,0 & 199,1 & 27,4 & 0,055 & 0,072 \\
\hline 8 & Rio Acre & 6,40 & 431,0 & 259,0 & 28,6 & 0,027 & * \\
\hline 9 & Rio Acre ( Casa Luis dos Anjos) & 6,86 & 480,0 & 287,0 & 27 & 0,030 & 0,088 \\
\hline 10 & Rio Acre (São João) & $\#$ & $\#$ & $\#$ & $\#$ & 0,037 & * \\
\hline 11 & Ig. Queimada (Rio Acre) & 6,83 & 422,0 & 252,0 & 25 & 0,032 & 0,100 \\
\hline 12 & Rio Acre & \# & \# & \# & \# & 0,073 & * \\
\hline 13 & Ig. Preto (Rio Acre) & 6,86 & 321,0 & 390,0 & 24,7 & 0,041 & 0,141 \\
\hline 14 & Rio Acre (Belmonte) & 6,91 & 485,0 & 276,0 & 27 & 0,041 & * \\
\hline 15 & Ig. Caiado(Cairala) (Rio Acre) & 6,86 & $1.151,0$ & 691,0 & 25,5 & 0,032 & 0,090 \\
\hline 16 & Rio Acre ( Colônia Nazaré) & 6,95 & 450,0 & 268,0 & 27 & 0,033 & * \\
\hline 17 & Ig. Nazaré (Rio Acre) & 6,94 & 410,0 & 246,0 & 24,2 & 0,046 & 0,103 \\
\hline 18 & Ig. Banana (Rio Acre) & 6,64 & 249,0 & 149,0 & 24,7 & 0,061 & 0,135 \\
\hline 19 & Ig. Banana (Rio Acre) & 6,67 & 261,0 & 155,4 & 26,2 & 0,031 & * \\
\hline
\end{tabular}




\section{ACTA AMAZONICA}

\begin{tabular}{|c|c|c|c|c|c|c|c|}
\hline \multirow{3}{*}{ Pontos } & \multirow{3}{*}{ Localização } & \multirow{2}{*}{\multicolumn{4}{|c|}{$\mathrm{H}_{2} \mathrm{O}$}} & \multicolumn{2}{|c|}{$\mathrm{Hg}$} \\
\hline & & & & & & \multirow[t]{2}{*}{$\begin{array}{l}\text { Sed. } \\
\mu \mathrm{g} \mathrm{g}^{-1}\end{array}$} & \multirow[t]{2}{*}{$\begin{array}{l}\text { Mat. Part. } \\
\qquad \mu \mathrm{g} \mathrm{g}^{-1}\end{array}$} \\
\hline & & $\mathrm{PH}$ & $\begin{array}{l}\text { Cond. } \\
\mu \mathrm{S} \mathrm{cm}^{-1}\end{array}$ & $\begin{array}{c}\text { STD } \\
\mathrm{mg} \mathrm{L}^{-1}\end{array}$ & $\underset{{ }^{\circ} \mathrm{C}}{\text { Temp }}$ & & \\
\hline 20 & Rio Acre & 6,73 & 328,0 & 197,0 & 26,8 & 0,065 & 0,072 \\
\hline 21 & Rio Acre ( montante Ig. Banana) & 6,69 & 340,0 & 204,0 & 27,4 & 0,018 & 0,071 \\
\hline 22 & Ig. S. Pedro (Rio Acre) & 6,73 & 398,0 & 239,0 & 25,1 & 0,078 & 0,136 \\
\hline 23 & Rio Acre ( jusante Ig. S. Pedro) & 6,67 & 378,0 & 225,0 & 24,9 & 0,077 & 0,071 \\
\hline 24 & Rio Acre ( Seringal. S. Pedro) & 6,70 & 377,0 & 226,0 & 24,5 & 0,132 & 0,075 \\
\hline 25 & Rio Acre ( local. Poção) & 6,67 & 380,0 & 229,0 & 25,3 & 0,037 & * \\
\hline 26 & Rio Acre ( local. Museu) & 6,68 & 385,0 & 213,0 & 25,7 & 0,067 & * \\
\hline 27 & Ig. S. Miguel (Rio Acre) & 6,67 & 281,0 & 168,4 & 24,7 & 0,074 & 0,220 \\
\hline 28 & Rio Acre ( Local.S. Miguel) & 6,62 & 385,0 & 233,0 & 26,1 & 0,050 & 0,080 \\
\hline 29 & Rio Acre (Local. Pedreira) & \# & \# & \# & \# & 0,040 & * \\
\hline 30 & Rio Acre (Local. Faz. do Bessa) & 6,70 & 439,0 & 264,0 & 26,7 & 0,051 & 0,077 \\
\hline 31 & Ig.Javari (Rio Acre) Peru/Bolívia & 6,63 & 347,0 & 211,0 & 25,4 & 0,065 & 0,068 \\
\hline 32 & Rio Acre & 6,70 & 471,0 & 284,0 & 27,7 & 0,088 & 0,067 \\
\hline \multicolumn{8}{|c|}{ RIO ACRE } \\
\hline & Média & 6,73 & 412,40 & 245,27 & 26,81 & 0,058 & 0,076 \\
\hline & Desvio padrão & 0,14 & 49,00 & 29,02 & 1,33 & 0,041 & 0,006 \\
\hline & Máximo & 6,95 & 485,00 & 287,00 & 29,00 & 0,184 & 0,088 \\
\hline & Mínimo & 6,40 & 328,00 & 197,00 & 24,50 & 0,018 & 0,067 \\
\hline \multicolumn{8}{|c|}{ AFLUENTES } \\
\hline & Média & 6,67 & 390,74 & 249,75 & 25,67 & 0,048 & 0,116 \\
\hline & Desvio padrão & 0,29 & 245,30 & 152,56 & 1,18 & 0,017 & 0,042 \\
\hline & Máximo & 6,94 & $1.151,00$ & 691,00 & 28,10 & 0,078 & 0,220 \\
\hline & Mínimo & 5,80 & 151,60 & 91,00 & 24,20 & 0,027 & 0,068 \\
\hline
\end{tabular}

pH - Potencial hidrogeniônico; Cond. - Condutividade elétrica; STD - Sólidos totais dissolvidos; Sed. - Semimento de fundo;

Mat.Part. - M aterial particulado suspenso; Temp. - Temperatura; \# - leituras não realizadas; * - material insufuciente para leitura

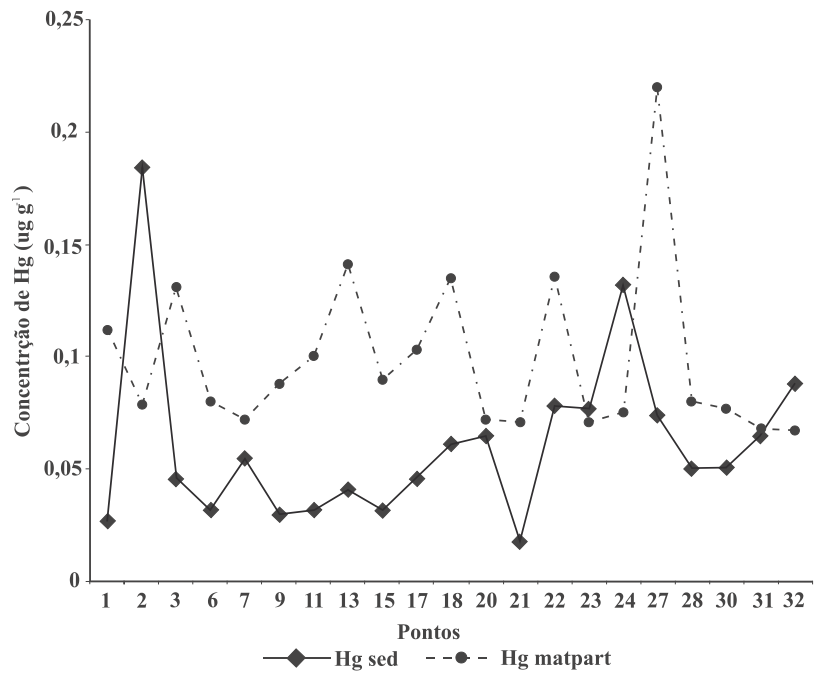

Figura 4 - Comparação entre os níveis de $\mathrm{Hg}$ em sedimentos e material particulado no rio Acre e seus afluentes.

\section{Parâmetros Físico-Químicos}

Neste estudo foram avaliadas variáveis físico-químicas $(\mathrm{pH}$, condutividade elétrica, sólidos totais dissolvidos e temperatura), medidas no campo, para verificar possíveis processos de fixação, transporte e transformação de $\mathrm{Hg}$ no ambiente aquático. Os valores de $\mathrm{pH}$ no rio Acre apresentaram uma variação discreta $(6,40$ a 6,95$)$, com média de 6,73, indicando um meio levemente ácido. Nos tributários do rio Acre os valores de $\mathrm{pH}$ variaram entre 5,80 e 6,94, com média de 6,67. As medidas de condutividade elétrica no rio Acre variaram de 328,00 a $485,00 \mu \mathrm{S} \mathrm{cm}^{-1}$, com média de $412,40 \mu \mathrm{S} \mathrm{cm}{ }^{-1}$, enquanto que nos tributários foram de 151,60 a 1.151,00 $\mu \mathrm{S} \mathrm{cm}^{-1}$. Somente um valor anômalo foi observado no Igarapé Caiado $\left(1.151,00 \mu \mathrm{S} \mathrm{cm}^{-1}\right)$.

Os valores de sólidos totais dissolvidos variaram de 197,00 a $287,00 \mathrm{mg} \mathrm{L}^{-1}$ no rio Acre, com média de 245,27 $\mathrm{mg} \mathrm{L}^{-1}$. Nos afluentes os valores, foram ligeiramente mais elevados, variando entre 91,00 e $691,00 \mathrm{mg} \mathrm{L}^{-1}$,com média 


\section{ACTA \\ AMAZONICA}

AVALIAÇÃO DA CONCENTRAÇÃO DE MERCÚRIO EM SEDIMENTOS

E MATERIAL PARTICULADO NO RIO ACRE, ESTADO DO ACRE, BRASIL de $249,75 \mathrm{mg} \mathrm{L}^{-1}$. A temperatura das águas no rio oscilou entre 24,50 e $29,00^{\circ} \mathrm{C}$, enquanto que nos igarapés variou de 24,20 e $28,10^{\circ} \mathrm{C}$.

Utilizando-se o Teste T-student, não foram observadas diferenças significativas nas médias de $\mathrm{pH}$ no rio Acre $(6,73)$ e de seus afluentes $(6,67)$; de condutividade 412,40 $\mu \mathrm{S} \mathrm{cm} \mathrm{cm}^{-1}$ no rio Acre e $397,66 \mu \mathrm{S} \mathrm{cm}^{-1}$ nos afluentes; sólidos totais dissolvidos $245,27 \mathrm{mg} \mathrm{L}^{-1}$ no rio Acre e $249,57 \mathrm{mg} \mathrm{L}$ ${ }^{1}$ nos afluentes e mercúrio nos sedimentos $0,058 \mu \mathrm{g} \mathrm{g}^{-1}$ no rio e $0,048 \mu \mathrm{g} \mathrm{g}^{-1}$ nos afluentes. Já a diferença entre a média de mercúrio em material particulado é significativa, visto que no rio Acre a média foi de $0,076 \pm 0,006 \mu \mathrm{g} \mathrm{g}^{-1}$, com intervalo de confiança de 0,071 - 0,080, enquanto que, nos afluentes a média encontrada foi de $0,116 \pm 0,042 \mu \mathrm{g}$ $\mathrm{g}^{-1}$, com intervalo de confiança $0,085-0,143$ e coeficiente de Pearson de 0,009 .

\section{DISCUSSÃO E CONCLUSÕES}

A determinação de $\mathrm{Hg}$ em sedimentos de fundo constitui-se num importante referencial para a avaliação da poluição de ambientes aquáticos por metais pesados (Silva et al., 1997). A determinação de $\mathrm{Hg}$ em material particulado suspenso representa um papel importante, considerando os aspectos relacionados ao transporte e mobilidade deste metal, visto que, a granulometria fina em suspensão pode adsorver e trocar íons com a solução transportá-los ao longo do corpo d'água e depositá-los em outros sítios de deposição (Cranston \& Buckley, 1972).

O mercúrio e outros metais pesados em ambientes aquáticos, ligam-se predominantemente ao material particulado em suspensão ( Forstner \& Muller, 1974; Forstner \& Patchinelam, 1976). Este aspecto foi observado no rio Acre e seus afluentes, onde os resultados mostram teores de $\mathrm{Hg}$ mais elevados no material particulado, quando comparado aos níveis nos sedimentos de fundo como mostrado na Figura. 4.

Não foram verificadas diferenças significativas entre as concentrações médias de $\mathrm{Hg}$ entre os sedimentos de fundo do rio Acre e de seus afluentes, porém observou-se uma significativa variação entre a média no material particulado em suspensão, possivelmente relacionada ao teor de matéria orgânica contida no material particulado ou ao tipo de argilominerais presentes.

As concentrações de $\mathrm{Hg}$ no rio Acre e seus afluentes, foram comparadas com as obtidas em outras drenagens, como por exemplo, no rio Rato, na Bacia do rio Tapajós, onde a garimpagem é desenvolvida em larga escala. Para o material particulado em suspensão as concentrações determinadas no rio Acre e seus afluentes foram semelhantes. Chama a atenção os níveis de $\mathrm{Hg}$ no material particulado em suspensão, com valores médios de 0,119 $\mu \mathrm{g} \mathrm{g} \mathrm{g}^{-1}$, encontrados no Rio Rato por Silva (1997). Nos afluentes do rio Acre foram observados valores médios da ordem de $0,116 \mu \mathrm{g} \mathrm{g}^{-1}$, mostrando a similaridade dos valores de $\mathrm{Hg}$ neste material de drenagens inseridas num contexto diferenciado na região amazônica.
Os valores médios em sedimentos de fundo do rio Acre e seus afluentes foram menores quando comparados com os obtidos no rio Rato $\left(0,230 \mu \mathrm{g} \mathrm{g}^{-1}\right)$ e seus afluentes $(1,60$ $\mu \mathrm{g} \mathrm{g}^{-1}$ ) (Silva, 1997). Estes valores relativamente elevados encontrados no Rio Rato são atribuídos às emissões localizadas de $\mathrm{Hg}$ próximas aos locais de extração de $\mathrm{Au}$, fato que não ocorre na bacia do rio Acre. Os teores de $\mathrm{Hg}$ em sedimentos de corrente do Rio Acre também estão abaixo da média das concentrações obtidas por Oliveira et al. (1999), em sedimentos da bacia do Rio Bento Gomes $(0,091$ $\left.\mu \mathrm{g} \mathrm{g}^{-1}\right)$, situado numa área sob influência de garimpos no município de Poconé-MT.

Comparativamente, os teores de $\mathrm{Hg}$ encontrados no rio Acre são também mais elevados que os teores médios de Hg obtidos por Castro e Silva et al. (1999), nos rios Cuiabá e Paraguai $\left(0,029 \mu \mathrm{g} \mathrm{g}^{-1}\right)$, na região do Pantanal, com pouca ou nenhuma atividade garimpeira, e também mais elevados que os teores médios de $\mathrm{Hg}$ em sedimentos encontrados por Silveira et al. (1999), no rio Madeira $\left(0,052 \mu \mathrm{g} \mathrm{g}^{-1}\right)$.

Os valores médios encontrados no rio Acre e seus afluentes estão abaixo do valor de "background" referido para os rios amazônicos não contaminados $<0,200 \mu \mathrm{g} \mathrm{g} \mathrm{g}^{-1}$ (Pfeiffer, et al.1989) e dentro da faixa estabelecida para rios amazônicos não contaminados $\left(0,05-0,28 \mu \mathrm{g} \mathrm{g}^{-1}\right)$, Lacerda et al. (1987).

Balogh et al. (1997) assinalou que o mercúrio dissolvido também é fortemente adsorvido nos sedimentos, nos sólidos em suspensão, incluindo a matéria orgânica, e nos óxidos e hidróxidos de ferro e manganês. Gagnon \& Fisher (1997) demonstraram que a força de ligação do mercúrio ao material particulado suspenso é muito grande e que a adsorção é pequena, menor que 10\%, ocorrendo em condições de $\mathrm{pH}$ ácido, menor que 5 . Este fato foi observado neste estudo, com valores da concentração média de $\mathrm{Hg}$ mais elevada no material particulado em suspensão e condições de $\mathrm{pH}$ variando entre 6,95 e 5,80.

Os resultados quanto a caracterização físico-química das águas do rio Acre e seus afluentes sugerem classificá-las como rios de águas brancas segundo Sioli, (1950); Junk \& Furch, (1980), ou seja, águas com pH neutro a levemente ácido, com condutividade elétrica moderada a alta, e elevada quantidade de material particulado em suspensão.

As características físico-químicas destas drenagens poderiam justificar eficientes processos de metilação, já que não são observados níveis elevados de mercúrio nos sedimentos e no material particulado suspenso, ou seja, os processos de metilação devem ocorrer de forma rápida, facilitada pela forma biodisponível do metal e pelas condições físico-químicas favoráveis nestes ambientes aquáticos.

Os baixos teores médios de $\mathrm{Hg}$ nos sedimentos e no material particulado, contrastam com os teores elevados encontrados na biota, principalmente nos peixes carnívoros, com uma concentração média de $1,287 \mu \mathrm{g} \mathrm{g}^{-1}$. Deste modo, sugerem eficientes mecanismos de transferência dos compartimentos abióticos para os bióticos (Silva et al. , 1999).

A possibilidade de os altos teores de $\mathrm{Hg}$ nos peixes 


\section{ACTA AMAZONICA}

do Rio Acre serem originadas por processos de metilação altamente eficientes em lagos formados pelos meandros deste e de outros rios de grande sinuosidade na região ocidental da Amazônia, durante as épocas de estiagem, formando condições lênticas, de alagados com grande quantidade de matéria orgânica submersa, é uma das hipóteses atualmente estudada pelos pesquisadores da SAMAM/IEC.

No entanto, apesar da ausência de evidências de atividades antropogênicas reconhecidamente emissoras de mercúrio, torna-se necessário a continuidade dos estudos para que se possa conhecer a origem (atmosférica e proveniente de outras áreas ou de origem proveniente do substrato geológico da região), e os mecanismos de biodisponibilidade do mercúrio na região.

\section{BIBLIOGRAFIA CITADA}

Akagi, H.; Malm, O.;Branches, F.J.P. 1996. Human exposure to mercury due to mining in the Amazon,Brasil- a review. Environmental Science, 3: 199-211.

Akagi, H.; Malm, O.; Branches, F. J. P.; Kinjo, Y.; Guimarães, J.R.D.; Oliveira, R.B.; Haraguchi, K.; Pfeffer, W. C.; Takizawa, Y.; Kato, H. 1995. Human exposure to mercury due to goldmining in the Tapajos River Basin, Amazon, Brasil: speciation of mercury in hair, blood and urine. Water, Air and Soil Pollution, 80: 85-94.

Balogh, S.J.; Meyer, M.L; Johnson, D.K. 1997. Mercury and suspended sediment loadings in the Lower Minnesota River. Environmental Science and Technology, 32(4): 456-462.

Barbosa, A.C.; Boischio, A. A.; East, G.A.; Ferrari, I.; Gonçalves, A.; Silva, P.R M.; Cruz, T.M.E. 1995. Mercury contamination in the Brazilian Amazon. Environmental and occupation aspects. Water, Air and Soil Pollution, 80: 109-121.

Brabo, E.; Santos, E.C.; Jesus, I.M.; Mascarenhas, A.F.S.; Faial, K.F. 1998. Verificação dos niveis de mercúrio no pescado consumido pela comunidade ribeirinha de Santana de Ituqui - Bacia do rio Amazonas - Santarém, PA: resultados preliminares. In: Anais do XXXIV Congresso Brasileiro de Medicina Tropical. Sociedade Brasileira de Medicina Tropical. Manaus, AM, p.40.

Brabo, E.S.; Santos, E.C.O.; Jesus, I.M.; Mascarenhas, A.F.S.; Faial, K.F. 1999. Níveis de mercúrio em peixes consumidos pela comunidade indígena de Sai Cinza na Reserva Munduruku, Município de Jacareacanga, Estado do Pará, Brasil. Cadernos de Saúde Pública, 15(2): 325-332.

Castro e Silva, E.; Oliveira, L.J.; Guimarães, J.R.D.; Hylander, L.D.;Pinto, F.N.; Mieli, M.;Malm, O.; Neves, R.P.; Stachiw, R.; Araújo, D.M. 1999. Total mercury in sediments from the Cuiabá and Paraguay river basins, Pantanal, Brazil. In: Book of Abstract of the $5^{\text {th }}$ International Conference-Mercury AS A Global Pollutant. Rio de Janeiro, RJ. p.515.

Costa, M.L.; Costa, W.A.M.; Santos, A.J.M., 1989. Exploração geoquímica para fosfatos no Estado do Acre. In: Anais do II Congresso Brasileiro de Geoquímica. Sociedade Brasileira de Geoquímica. Rio de Janeiro, RJ, p. 29-37.
Cranston, R.E.; Buckley, D.E. 1972. Mercury pathways in a river and estuary. Environmental Science and Technology, 6(3): 274-278.

FIBGE (Fundação Instituto Brasileiro de Geografia e Estatística) 1990. Projeto zoneamento das potencialidades dos recursos naturais da Amazônia Legal. Rio de Janeiro. 212pp.

Forstner, U.; Patchineelam, S.R. 1976. Binung und remobilizierung von Schwermetallen in Fluvitilen. Chemikerzeitung. 49-57.

Forstner, U.; Muller, G., 1974. Schwermetalle in Flussen und Seen als ausdruck der umweltverschumuzung. Springer-Verlag. New York, 225pp.

Fostier, A.H.; Oliveira, S.M.B.; Guimarães, J.R.D.; Forti, M.C.; Melfi, A.J.; Boulet, R.; Favaro, D.I.T.; Krug, F.J. 1999. Mercury accumulation in natural forested amazonian soils. In: Book of Abstract of the $5^{\text {th }}$ International Conference-Mercury As A Global Pollutant. Rio de Janeiro, RJ, p.557.

Gagnon,C.; Fisher, N.S. 1997. Bioavailability of sediments-bound methyl and inorganic mercury to a marine bivalve. Environmental Science and Technology. 31(4): 993-998.

Hacon, S.; Rochedo, E.R.; Campos, R.; Rosales, G.; Lacerda, L.D. 1997. Risk assessment of mercury of in Alta Floresta. Amazon Basin-Brazil. Water, Air and Soil Pollution, 97: 91-105.

Jesus, I.M.; Santos, E.C., Brabo, E.S.; Loureiro, E.C.B.; Mascarenhas, A.F.S. 1998. Exposição ao mercúrio em trabalhadores das lojas de compra e venda de ouro nas cidades de Santarém e Itaituba,PA. In: Anais do XXXIV Congresso Brasileiro de Medicina Tropical. Sociedade Brasileira de Medicina Tropical. Manaus, AM, p.41.

Junk, W.J.; Furch, K. 1980. Química da água e macrofitas aquáticas de rios e igarapés na Bacia Amazônica e nas áreas adjacentes. Parte I. Acta Amazonica, 10: 611-633

Lacerda, L.D.; Pfeiffer, W.C.; Silveira, E.G; Bastos, W.R. Souza; C.M.M. 1987. Contaminação por mercúrio na Amazônia; análise preliminar do rio Madeira, RO. In: Anais do II Congresso Brasileiro de Geoquímica. Sociedade Brasileira de Geoquímica. Rio de Janeiro, RJ, p.295-299.

Lebel, J.; Roulet, M.; Mergler, D.; Lucotte, M.; Larribe, F. 1997. Fish diet and exposure in a riparian Amazon population. Water, Air and Soil Pollution, 97: 31-44.

Malm, O. 1998. Gold mining as a source of mercury exposure in the Brazilian Amazon. Environmental Research, 77: 73-78.

Malm, O.; Branches, F.J.P; Akagi, H.; Castro, M.B.; Pfeiffer, W.C.; Harada, M.; Bastos, W.R.; Kato, H. 1995. Mercury and methylmercury in fish and human hair from the Tapajós River Basin, Brazil. The Science of the Total Environment, 175: 141-150.

Malm, O.; Pfeiffer, W.C.; Souza, C.M.M.; Reuter, R. 1990. Mercury pollution due to gold mining in the Madeira River Basin, Brazil. Ambios, 19: 11-15.

Martinelli, L.A.; Ferreira, L.R.; Forsberg, B.R.; Victoria, R.L. 1988. Mercury contamination in the Amazon: a gold rush consequence. Ambios, 17: 252-254.

Mascarenhas, A. F.S.; Brabo, E.S.; Santos, E.C., Jesus, I.M.; Faial, K. F. 1998. Níveis de mercúrio em peixes na bacia hidrográfica do Tapajós, Jacareacanga - Itaituba Pará: resultados preliminares. In: Anais do XXXIV Congresso Brasileiro de Medicina Tropical. Sociedade Brasileira de Medicina Tropical. Manaus, AM, p.41. 
Oliveira, L.. J.; Hylander, L. D.; Barros, A.J.P.; Singulane, L.M.C.; Laet, S.M.; Barreto, L.B.; Silva, G.D.; Araújo, S. A. 1999. Monitoring of heavy metals in thr Bento Gomes river basin, Poconé, Mato Grosso, Brasil. In: Book of Abstract of the $5^{\text {th }}$ International ConferenceMercury As A Global Pollutant. Rio de Janeiro, RJ, p. 522.

Pfeiffer, W.C.; Lacerda, L.D. 1988. Mercury Imputs into the Amazon Region, Brazil. Environmental Technology Letters, 9: 325-330.

Pfeiffer, W.C.; Lacerda, L.D.; Souza, C.M.M.; Silveira, E.; Bastos, W.R. 1989. Mercury Concentrations in inland waters of gold mining areas in Rondônia, Brazil. The Science of the Total Environment, (87/88): 233-240.

Ribeiro Jr. M.G.; Silva Filho, E.V.; Souza, M.; Lacerda, L.D. 1999. Mercury burden in soils from Central Amazon. In: Book of Abstract of the $5^{\text {th }}$ International Conference Mercury As A Global Pollutant. Rio de Janeiro, RJ, p. 558.

Rodrigues, R.M; Mascarenhas, A.F.S.; Ichiara, A.H.; Souza, T.M.C.; Bidone, E.D.; Bellia, V.; Hacon, S.; Silva, A.R.B.; Braga, J.B.; Filho, B.S. 1994. Estudo dos impactos ambientais decorrentes do extrativismo mineral e poluição mercurial no Tapajós - prédiagnóstico. Série Tecnologia Ambiental, CETEM/CNPq. Rio de Janeiro, RJ, 220pp.

Roulet, M.; Lucotte, M., 1995. Geochemistry of mercury in prestine and flooded ferrolitic soils of a tropical rain forest in French Guiana, South America. Water, Air and Soil Pollution, 80: 1079-1088.

Roulet, M.; Lucotte, M.; Saint-Aubin, S.; Heault, I.; Farella, I.; Silva, E.J.; Dezencourt, J.; Souza Passos, C.J.; Soares, G.S.; Guimarães, J.R.; Mergler, D.; Amorim, M. 1998. The geochemistry of mercury in central amazon soils developed on the Alter do Chão formation of the lower Tapajós river valley, Pará State, Brazil. The Science of the Total Environment , 223(1): 1-24.

Santos, E.C., Jesus, I.M.; Brabo, E.S.; Loureiro, E.C.B.: Weirich, J.; Mascarenhas, A. F.S.;. 1998. Níveis de mercúrio em tecido capilar na comunidade indígena de Sai Cinza, Reserva Munduruku, Jacareacanga- Pará. In: Anais do XXXIV Congresso Brasileiro de Medicina Tropical. Sociedade Brasileira de Medicina Tropical. Manaus, AM, p.40.

Santos, E.O. 1993. Garimpagem e Saúde. In: Armin, M.; Rehaag, R. (Eds.). Conseqüências da Garimpagem no Âmbito Social e Ambiental da Amazônia. CEJUP, Belém, Pará, p. 36-37.

Santos, E. C. O.; Jesus, I. M.; Brabo, E. S.; Câmara, V. M.; Loureiro, E. C. B.; Mascarenhas, A. F. S.; Faial, K. F. F.; Silva, A. P.; Silva, R. U.; Luiz, R. R.; Higuchi, H. 2002. Exposure to mercury in the urban population of Rio Branco city, State of Acre, Brazil. Bulletin of Environmental Contamination and Toxicology, New York, 69(3): 314-319.

Silva, A.P. 1993a. As diversas formas de garimpo de ouro, suas emissões de mercúrio e mecanismos de dispersão nos diversos compartimentos ambientais. In: Armin, M.; Rehaag, R. (Eds.). Conseqüências da Garimpagem no Âmbito Social e Ambiental da Amazônia. CEJUP, Belém, Pará. p.48-56.

Silva, A.P. 1993b. Coleta de amostras em solos, sedimentos e águas de ambientes impactados por mercúrio para monitoramento ambiental. In: Câmara, V.M.(ed.). Mercúrio em áreas de garimpos de ouro. ECO/OPS. México. p. 99- 105.
Silva, A.P. 1996. Mercúrio em ambientes aquáticos de Poconé-MT. Tese de Doutorado, Instituto de Geociências, Universidade de São Paulo, São Paulo, SP. 123pp.

Silva, A.P. 1997. Projeto Itaituba: programa de ciência e tecnologia ambiental. Série Tecnologia Ambiental. CETEM/CNPq. Rio de Janeiro, RJ, 66pp.

Silva, A.P.; Ramos, A.L.A.; Bastos, W.R. 1993. Mercury in particulated suspended sediments: a new sampling methodology. In: Proceedings of Perspectives for Environmental Geochemistry in Topical Countries. Niterói, Rio de Janeiro. p. 440-451.

Silva, A P.; Santos, E.C.; Brabo, E.S.; Câmara, V.M.; Mascarenhas, A.F.S.; Jesus, I.M.; Faial, K.R.F., Loureiro E.C.B. 1999. Contribuição ao estudo da poluição por mercúrio no Estado do Acre. Revista Saúde e Ambiente, 31(1/2): 34-42.

Silveira, E.G.; Bastos, W.R.; Malm, O.; Bonotto, D.M. 1999. Characterization of Mercury in Bottom sediments and rocks in Madeira river (RO), between Teotônio and Santo Antônio falls. In: Book of Abstract of the $5^{\text {th }}$ International ConferenceMercury As A Global Pollutant. Rio de Janeiro. p. 544 .

Sioli, H. 1950. Das wasser in Amazonasgebeit. Forschung Fortchrift, $26: 274-280$.

Veiga, M. M.; Meech, J.A. ; Onate. N., 1994. Deflorestation: A major sources of mercury pollution in the Amazon. Nature, 368: 816-817.

Zeidemann, V.K.; Forsberg, B.R.; Roulet, M.; Lucotte, M.; Jardim, W.F. 1999. The geochemistry of mercury in soils of the Negro River Basin Brazilian Amazon and its role in the regional mercury cycle. In: Book of Abstract of the $5^{\text {th }}$ International ConferenceMercury As A Global Pollutant. Rio de Janeiro. p. 561.
RECEBIDO EM 17/04/2001
ACEITO EM 26/11/2003 